\title{
EL PRETENDIDO EFECTO RETROACTIVO DE LA CONDICIÓN CUMPLIDA
}

\section{PATRICIO LAZO*}

RESUMEN: El objeto del estudio es el examen de las fuentes romanas, en cuanto se pronuncian acerca de si la condición cumplida pudo o no tener efecto retroactivo. En efecto, en tanto en algunas fuentes se afirma que sí lo tuvo, en otras, tal efecto es sencillamente negado. Para el examen, el A. se vale de la premisa metodológica según la cual los juristas romanos suelen volver sobre los casos tratados por juristas precedentes, para así confirmar la doctrina anterior, o bien, para establecer variantes en la solución de los problemas. Desde esta perspectiva, se analiza el contenido de varios de los fragmentos referidos al mismo problema, intentando establecer conexiones internas entre cada uno de ellos, para así sugerir el verdadero alcance de los fragmentos que afirman la retroactividad de la condición.

Palabras Clave: Condición, retroactividad, casuismo jurisprudencial, obligaciones.

ABSTRACT: The topic of this article is the study of Roman sources of law, to the extent that they determine whether the condition which has been fulfilled could have had, or no, a retroactive effect, since some of those sources answer "yes, there was such a retroactive effect", while others simply deny it. In examining those texts, the author assumes the methodological premise according to which Roman jurists were used to review the cases as decided by earlier jurists, in order, either to confirm the old jurisprudence, or to establish new and varying solutions to the problems. In that perspective, the content of several fragments which refer to the same problems are analyzed so that internal connections between each of them can be uncovered and the true scope of the fragments which say the condition is retroactive can be determined.

Key words: condition, retroactivity, caselaw, obligations.

El problema de si en el derecho romano la condición cumplida tuvo o no efecto retroactivo no es nuevo; más bien puede decirse que, cuando menos, en la manualística es un tema que no suscita debate, no obstante que no ha faltado quien haya alegado la persistente falta de claridad acerca del punto ${ }^{1}$. Quizá esta sola constatación sea razón suficiente para volver sobre el tema. En mi opinión, es necesario revisar los resultados con los que, sobre esta materia, contamos; además, creo que la utilización de una perspectiva metodológica diferente puede servir para llegar a resultados en sí mismos novedosos, con independencia de si la revisión arroja resultados diferentes a los conocidos.

\footnotetext{
* Profesor de Derecho Romano de la Universidad de Antofagasta.

${ }^{1}$ Vid. TAlamanca (1990) p. 258 sostiene que, a pesar de fragmentos como los del Gai. 3, 146 y D. 18, 1, 8 pr., testimonian que los juristas romanos consideraron que, cumplida la condición, los efectos debían considerarse producidos a partir del momento en que se había concluido el negocio, puede dudarse del efectivo alcance dogmático de estas declaraciones. Con menos dudas, KASER (1971) p. 256 afirma que los juristas romanos no atribuyeron con carácter general efecto retroactivo a la condición cumplida. En el mismo sentido, MITTEIS (1994) p. 175, se inclinaba por la negativa, no obstante considerar que existían algunos casos en los que, a simple vista, parecía darse este efecto retroactivo.
} 
Este trabajo tiene su origen en un estudio acerca de la doctrina del riesgo de la compraventa en un texto de Paulo: D. 18, 6, $8 \mathrm{pr}^{2}$. En este pasaje, conocido porque en él se sistematiza como en ningún otro la doctrina del periculum emptoris, el jurista se coloca ante la siguiente hipótesis: si la compraventa se ha sometido a condición y esta se cumple, el riesgo es del comprador (quod si sub condicione res venierit, si quidem defecerit condicio, nulla est emptio, sicuti nec stipulatio: quod si exstiterit, Proculus et Octavenus emptoris esse periculum aiunt: idem Pomponius libro nono probat). Queda un poco en el aire la elucidación de si la cosa ha debido perecer antes o después del cumplimiento de la condición, pero es lógico pensar que se trata de lo primero, es decir, del perecimiento anterior al cumplimiento de la condición. Ante la pregunta de por qué ello es así, Seckel y Levy ${ }^{3}$ argumentan que el comprador soporta el riesgo en el evento mencionado, en razón del efecto retroactivo de la compraventa. Se trataría, a decir de estos autores, de una doctrina de origen proculeyano, que habría terminado por ser la dominante. Los autores citados, además, conjeturan que Pomponio, de filiación sabiniana, habría sostenido, precisamente, la doctrina contraria. Por las razones que expongo en otro trabajo ${ }^{4}$, no creo que una conjetura que se aleja tanto del texto represente una posibilidad cierta.

El pasaje continúa con una referencia a la hipótesis de la muerte del comprador o del vendedor antes del cumplimiento de la condición. No se trata, pues, de un caso de riesgo de la cosa. Pero es interesante, por cuanto su argumento trasluce el mentado efecto retroactivo de la condición. En efecto, Paulo señala a este respecto que, en caso de morir el vendedor o el comprador, al cumplirse la condición, la obligación se radicará en los herederos, como si esta hubiese sido contraído en el pasado (condicio, heredes quoque

\footnotetext{
${ }^{2}$ D. 18, 6, 8 pr.: Paul. 33 ad ed. D.18.6.8pr.: Necessario sciendum est, quando perfecta sit emptio: tunc enim sciemus, cuius periculum sit: nam perfecta emptione periculum ad emptorem respiciet. et si id quod venierit appareat quid quale quantum sit, sit et pretium, et pure venit, perfecta est emptio: quod si sub condicione res venierit, si quidem defecerit condicio, nulla est emptio, sicuti nec stipulatio: quod si exstiterit, Proculus et Octavenus emptoris esse periculum aiunt: idem Pomponius libro nono probat. quod si pendente condicione emptor vel venditor decesserit, constat, si exstiterit condicio, heredes quoque obligatos esse quasi iam contracta emptione in praeteritum. quod si pendente condicione res tradita sit, emptor non poterit eam usucapere pro emptore. et quod pretii solutum est repetetur et fructus medii temporis venditoris sunt (sicuti stipulationes et legata condicionalia peremuntur), si pendente condicione res exstincta fuerit: sane si exstet res, licet deterior effecta, potest dici esse damnum emptoris. [Es necesario saber cuándo la compra se encuentra perfecta: y entonces sabremos de quién es el riesgo, pues una vez perfecta la compra, el riesgo pertenecerá al comprador. Y si consta claramente lo que se vendió, la calidad, la cantidad y el precio, y se hubiese vendido sin condición, se perfecciona la compra; pero si se hubiese vendido bajo condición y esta se frustrare, la compra es nula, como también la estipulación. Pero si la condición se hubiese cumplido, Próculo y Octaveno dicen que el riesgo es del comprador y así lo aprueba también Pomponio. Si, estando pendiente la condición, hubiera muerto el comprador, o el vendedor, consta que, si la condición se cumpliese, también habrán de hallarse obligados los herederos, como si ya hubiese sido contraída a compra en el pasado. Si la cosa ha sido entregada mientras la condición estaba pendiente, el comprador no podrá usucapir por compra, lo que se pagó del precio podrá repetirse, y los frutos del tiempo intermedio son del vendedor; <las compras>, así como las estipulaciones y legados condicionales, se extinguirán si pendiente la condición, la cosa se hubiese extinguido; pero, si la cosa permanece, aunque se hubiese deteriorado, puede decirse que el perjuicio es del comprador.

3 SeCKel y LEVY (1927) p. 165.

${ }^{4}$ LAZO (2005).
} 
obligatos esse quasi iam contracta emptione in praeteritum). De las dos hipótesis con las que continúa el pasaje (perecimiento y deterioro de la cosa antes del cumplimiento de la condición), la primera (perecimiento) desmiente el efecto retroactivo de la condición, en tanto que la segunda más bien la aplica.

Este testimonio indicaría que, salvo uno de los casos, la regla general en el derecho romano clásico habría sido la del efecto retroactivo de la condición 5 . A partir de esta afirmación el objeto de este trabajo será indagar acerca de si los testimonios jurisprudenciales, desde los tiempos de la República, han sido unívocos en este sentido. Para mi análisis me centraré en textos relativos a la estipulación, en los cuales se aborda el caso del perecimiento de una cosa pendiente la condición que ha sido establecida para la efectividad de dicha estipulación. Este interés en la casuística sobre la estipulación condicional se relaciona con el estudio del efecto de la condición cumplida en la compraventa y puede ser justificado sobre la siguiente base: de una parte, la información que sobre esta cuestión ofrece el tema de la estipulación se coloca en oposición a lo que ocurre con los testimonios relativos compraventa y sobre idéntica materia, en que tal variedad resulta inexistente. Por otra parte, pienso que resulta razonable creer que, a pesar de no contar los juristas romanos con una teoría del negocio jurídico, tal como contamos en el derecho moderno, no deberían haber existido diferencias sustanciales al aplicar los efectos de la condición cumplida y, en particular, de los efectos producidos por el perecimiento de la cosa estipulada antes del cumplimiento de la condición. O mejor dicho, ningún testimonio existe que pueda probar que para la jurisprudencia romana, la condición y sus efectos estaba sometida a un régimen diferente en la compraventa que en la estipulación. Ello me anima a pensar que la situación debía haber sido precisamente la inversa, esto es, que frente a similares supuestos de hecho, la solución debió haber sido, en sustancia, la misma tanto para el caso de la estipulación que para el caso de la compraventa.

Para efectuar mi análisis, me valdré, a modo de premisa metodológica, de los resultados alcanzados por la investigación de García Garrido acerca del método de los juristas. Como se sabe, como productos de varios trabajos, el romanista español muestra un aspecto muy importante del trabajo de los juristas, como lo es la utilización de casos, reales o ficticios. A juicio de nuestro autor, los juristas romanos acostumbraban a trabajar sobre casos ya resueltos por otros juristas, con un doble propósito: por una parte, se servían de las soluciones dadas por los otros juristas a los casos y las utilizaban como guías de las soluciones que ellos mismos daban, originando lo que García Garrido llama casos-guía. En esta labor se servían principalmente, de la analogía. Por otra parte, sobre la base de estas soluciones introducían variantes, que significaban, en buenas cuentas, detener la vigencia de la solución anterior e introducir una solución innovadora ${ }^{6}$.

\footnotetext{
5 Sin embargo, KASER (1971) p. 257 n. 46, atribuye a la doctrina posclásica la generalización del efecto retroactivo, tomando como testimonio de todo ello, precisamente, D.18, 6, 8 pr.

${ }^{6}$ Una buena síntesis de los principales resultados de varios trabajos del autor sobre este aspecto de la labor de los juristas romanos puede verse en los siguientes trabajos: GARCÍA GARRIDO (1987) pp. 517-537; GARCÍA GARRIDO (1988) pp. 249-261; GARCÍA GARRIDO (2004b) pp. 25 ss.
} 


\title{
1. SERVIO Y GAYO
}

El análisis de casos comienza con uno planteado por Gayo. La razón para colocar al inicio de nuestro examen un fragmento perteneciente a un jurista de fines del s. II $\mathrm{d}$. C. se encuentra en el hecho de que a través de este pasaje se nos informa de un responsum de Servio Sulpicio sobre la misma cuestión. Ello nos permite establecer la jurisprudencia republicana como aquella en cuyo seno se planteó la primera decisión a este respecto); de ahí en adelante, como se verá, los supuestos del caso, fueron similares en los juristas posteriores. Incluso los problemas acerca de los cuales se pronunciaban guardaban un alto grado de correspondencia: A su turno, las diferencias, provenientes de variantes introducidas por los juristas, tienden, en su mayoría, a explicar las diferencias de opinión que pueden constatarse.

\begin{abstract}
Gai. 3.179
Quod autem diximus, si condicio adiciatur, novationem fieri, sic intellegi oportet, ut ita dicamus factam novationem, si condicio exstiterit; alioquin si defecerit, durat prior obligatio. sed videamus, num is, qui eo nomine agat, doli mali aut pacti conventi exceptione possit summoveri, quia videtur inter eos id actum, ut ita ea res peteretur, si posterioris stipulationis exstiterit condicio. Servius tamen Sulpicius existimavit statim et pendente condicione novationem fieri, et si defecerit condicio, ex neutra causa agi posse et eo modo rem perire; qui consequenter et illud respondit, si quis is, quod sibi L. Titius deberet, a servo fuerit stipulatus, novationem fieri et rem perire, quia cum servo agi non posset. sed in utroque casu alio iure utimur. nec magis his casibus novatio fit, quam si id, quod tu mibi debeas, a peregrino, cum quo sponsus communio non est, SPONDES verbo estipulatus $\operatorname{sim}^{7}$.
\end{abstract}

Gayo se refiere a un negocio -que llamaré "negocio superpuesto"- al cual se añade una condición. El hecho de que este negocio viene a superponerse a otro, que en lo sucesivo llamaré "negocio originario", puede extraerse sin gran dificultad de la referencia de Gayo a la novación. Respecto al negocio superpuesto, el comienzo del fragmento no permite determinar de cuál se trata; sin embargo, la parte final del texto parece dejar suficientemente claro que Gayo, cuando menos pensaba en una estipulación. Pensar que el negocio originario lo constituía también una estipulación, no es, tomando en cuenta

\footnotetext{
${ }^{7}$ Lo que hemos dicho de que si se añade una condición hay novación, hay que entenderlo en el sentido de que se cumpla esta condición, pues si no se cumple dura la obligación antigua. Sin embargo, hay que ver si el acreedor que reclama en este supuesto no puede ser rechazado por una excepción de dolo malo o de pacto ajustado, pues, efectivamente, las partes parecen haber querido que el objeto de la obligación fuera exigible solamente en caso de cumplirse la condición. En efecto, Servio Sulpicio era de la opinión de que la novación tenía efectos desde luego, aunque estuviera pendiente la condición, y que, en caso de frustrarse esta, no quedaba acción ninguna y se perdía la cosa. De acuerdo con esto, respondió que también se hacía novación y se perdía la cosa cuando uno estipula de un esclavo lo que le debe L. Ticio, ya que no tiene acción contra el esclavo. Pero en ambos supuestos seguimos otros principios jurídicos: en ningún caso de estos hay novación, así como tampoco en el caso de que, al estipular de un extranjero lo que me debes, se emplee la fórmula ¿TE COMPROMETES?, que aquel no puede usar.
} 
este dato, descaminado. Debe también hacerse notar que, en este punto, interesa a Gayo la cuestión relativa al momento en que puede decirse que la obligación anterior se encuentra novada; por ello aclara que, en el caso de haberse convenido una condición, la novación se producirá solo cuando la condición se cumpla ${ }^{8}$. Esta afirmación sirve a Gayo para preguntarse qué ocurriría en caso de reclamar el acreedor la obligación nacida del negocio originario. En mi opinión, Gayo se refiere al hecho de que el acreedor haga uso de la acción nacida del negocio originario, probablemente la condictio triticaria ${ }^{9}$. Es obvio que no pudo haberse referido a la obligación nacida del negocio superpuesto (estipulación condicional), ya que al no ser exigible la obligación, a causa de haber fallado la condición si (alioquin defecerit) y, por tanto, por no haber nacido jamás la obligación, de ningún modo habría dispuesto el acreedor de esta acción; además, visto de esta manera, la pregunta que se hace Gayo es lógica desde todo punto de vista: si, como él sostiene, la estipulación condicional no tiene efecto novatorio sino hasta verificarse el cumplimiento de la condición, luego no se ha extinguido aún la obligación nacida del negocio anterior y, en este sentido, es lógico preguntarse si la acción para reclamar esta obligación puede entablarse, una vez constatado el hecho de que la condición de la cual dependía el nacimiento de la nueva obligación ha fallado. La respuesta de Gayo, frente a esta pretensión del acreedor es negativa: con fundamento en el hecho de que al pactar la condición, puede presumirse que las partes han querido que el objeto fuese exigible solo una vez cumplida la condición, afirma Gayo que entonces cabría contra la acción ejercitada por el acreedor, o bien una exceptio doli, o bien una exceptio pacti conventi ${ }^{10}$. En la última parte del fragmento, refiere Gayo la opinión de Servio Sulpicio y ello tiene una importancia no menor. Por un lado, según Servio Sulpicio, el efecto novatorio del que venía hablando Gayo se produce nada más concluida la segunda estipulación, por lo que no se hace necesario el cumplimiento de la condición (existimavit statim et pendente condicione novationem fieri). Por otro lado, afirma Servio Sulpicio que si la cosa perece antes del cumplimiento de la condición, el acreedor carece de acción para exigir el cumplimiento, tanto de esta obligación, como de la anterior (ex neutra causa agi posse et eo modo rem perire). Finalmente, Gayo se reafirma en su convicción de que no hay novación con la sola conclusión de la estipulación condicional.

Se constata, pues, la existencia de un caso que fue, primeramente, resuelto por Servio Sulpicio y, más tarde, también Gayo. Como se verá, hubo entre estos dos juristas varios otros que trataron del mismo caso, aunque introduciendo variantes. Para los efectos de realizar la comparación casuística que me propongo llevar a cabo, expondré sintéticamente tres grupos de cuestiones que en cada caso son tratadas, de modo exhibir con claridad las diferencias entre uno y otro jurista. Estos grupos de cuestiones son los siguientes: a) supuestos del caso; b) problemas planteados al jurista; c) respuesta del jurista.

\footnotetext{
${ }^{8}$ Lo cual, según KASER (1971) p. 648 n. 18, corresponde al régimen clásico.

9 Al tratarse de una stipulatio certae rei, la acción correspondiente debe ser la condictio triticaria. Vid. Castresana Herrero (1994) p. 448.

10 Sobre lo cual vid. KASER (1971) p. 642 n. 12.
} 
Expuestos sintéticamente los resultados que se obtienen del caso tratado por Servio Sulpicio, de acuerdo con los criterios antes expresados, se obtiene lo siguiente. En cuanto a los supuestos, estos son tres: i) el debitum consistía en un esclavo; ii) la estipulación que se superpone es condicional; iii) la muerte del esclavo se produce antes del cumplimiento de la condición. Los problemas planteados a Servio Sulpicio serían los siguientes: i) la elucidación del momento en el cual se produce el efecto novatorio de la nueva estipulación; ii) los efectos de la muerte del esclavo, antes del cumplimiento de la condición, de cara a la acción ejercitable por el acreedor. Finalmente, la respuesta de Servio a ambas cuestiones es la siguiente: i) el efecto novatorio se produce como efecto de la conclusión de la nueva estipulatoria, aunque la condición no se haya cumplido aún; luego, la obligación anterior queda extinguida; ii) de producirse la muerte del esclavo antes de cumplimiento de la condición, queda el acreedor en la situación de no poder ejercitar ni la acción correspondiente al negocio originario ni la acción nacida del negocio superpuesto, ya que, al no haberse cumplido la condición no pudo nacer la obligación ni, por tanto, la acción con la cual reclamarla.

Tratándose de Gayo, los resultados pueden sintetizarse así: tanto los supuestos, como los problemas planteados al jurista son los mismos. A su turno, las diferencias se producen de cara a la respuesta de Gayo, frente a tales problemas: i) la novación se produce con el cumplimiento de la condición; ii) la muerte del esclavo antes del cumplimiento de la condición no impide que, eventualmente, pueda el acreedor hacer uso de la acción nacida del negocio originario. Pero concede Gayo al deudor dos excepciones: la de dolo y la de pacto ajustado, las cuales podrá oponer a la acción nacida del negocio originario. El fundamento para la concesión de estas excepciones lo ve Gayo en la interpretación de la estipulación condicional, como expresión de la voluntad de las partes de que el esclavo solo fuese entregado si se cumplía una condición.

Todo lo anterior lleva forzosamente a la conclusión de que entre este arco temporal que va entre el s. I a. C. al s. II d. C. se produce una importante modificación en cuanto a la determinación del momento en el cual se produce el efecto novatorio. Desde el punto de vista de los efectos de la muerte del esclavo, las opiniones de ambos juristas dejan sin perspectivas de éxito al acreedor. La diferencia está en que mientras Servio ve extinguida su acción por el solo hecho de la conclusión de la estipulación, Gayo no declara tal acción extinguida, sino que concede excepciones al deudor. Este modo de exhibir la opinión de Servio Sulpicio y la suya propia, creo que permiten aventurar la existencia de una soterrada (en las fuentes) controversia jurisprudencial. De la opinión de Servio Sulpicio, al parecer minoritaria ${ }^{11}$, cabe deducir que, cuando menos para una parte de la jurisprudencia romana, la estipulación condicional representaba un riesgo para el acreedor, ya que, por una parte, extinguía la obligación preexistente y, por otra, cargaba con el riesgo del perecimiento de la cosa debida, antes del cumplimiento de la condición, al acreedor.

11 Sobre la prevalencia de la opinión gayana, D’ORS (1986) p. 494. 


\section{SABINO, PAULO Y ULPIANO}

Me interesa a continuación mostrar cómo un pasaje de Paulo, en que refiere un solución de Sabino, puede ilustrar acerca de la fortuna que tuvo la doctrina sentada por Servio en un jurista, como Sabino, inmediatamente posterior. A su turno, un pasaje extraído de una obra de Ulpiano tiene la virtud de mostrar cómo el caso tratado por Sabino, es recogido por un jurista tardoclásico, quien acoge la doctrina sabiniana.

El primer caso, como se dijo, es uno resuelto por Sabino que es citado por Paulo. Debe advertirse que no puede considerarse que este caso dependa del de Servio Sulpicio, pero sí que trata de cuestiones muy relacionadas con aquellas de las que este se hacía cargo. Mi interés es mostrar cómo la doctrina sabiniana sobre la expedibilidad de la acción nacida de una estipulación y las condiciones bajo las cuales se negaba, tuvo también incidencia en el parecer de los juristas que se ocuparon del caso propuesto por Servio Sulpicio.

D. 45.1.83.7 (Paul. 72 ad ed.): Stichum, qui decessit, si stipuler, si quidem condici etiam mortuus potuit, ut furi, utiliter me stipulatum Sabinus ait: si vero ex aliis causis, inutiliter, quia et si deberetur, morte promissor liberetur. idem ergo diceret et si mora facta defunctum stipularer ${ }^{12}$.

El supuesto sobre la cual responderá Sabino es el siguiente: se ha estipulado un esclavo que ha muerto con posterioridad a la conclusión de la estipulación. El problema que se plantea a Sabino es acerca de la eficacia de la acción nacida de la estipulación, esto es, acerca de la posibilidad que tiene el estipulante de ejercitar eficazmente la acción negocial para hacer efectiva la prestación del promitente (arg. utiliter me stipulatum). Su responsum establece que se habrá estipulado eficazmente si el esclavo hubiese podido ser reclamado mediante la condictio, aunque hubiese muerto, tal como ocurre con el ladrón, uno de los casos en que la condictio es procedente (si quidem condici etiam mortuus potuit, ut furi, utiliter me stipulatum). Pero si el esclavo con anterioridad a la estipulación no hubiese sido exigible por medio de la condictio, el promitente queda liberado, en razón de la muerte del esclavo. El texto se cierra con una extensión de esta ratio decidendi al caso en que el esclavo hubiese sido anteriormente debido y se hubiese encontrado en mora. Si bajo estas circunstancias, se estipula el esclavo, entonces nuevamente debemos entender que el acreedor ha estipulado eficazmente.

Esta misma doctrina la reconocemos en un pasaje ulpianeo:

12 Si estipulo el esclavo Estico, que ha muerto, dice Sabino que la estipulación es válida siempre que aquel esclavo muerto pueda deberse por la condicción, como ocurre con el ladrón, pero que en los otros casos es inválida, ya que el promitente, aún habiéndose hecho deudor del esclavo, queda liberado por la muerte del mismo. Y lo mismo diría, por consiguiente, si se estipula el esclavo muerto de quien ha incurrido en mora en su obligación de darme aquel esclavo. 
D.45.1.82.1 (Ulp. 78 ad ed.):

Si post moram promissoris homo decesserit, tenetur nihilo minus, proinde ac si homo viveret ${ }^{13}$.

Ulpiano, en forma muy escueta, informa del supuesto de este caso: se ha estipulado la entrega de un esclavo y, encontrándose en mora el promitente, el esclavo muere. Según Ulpiano, el promitente mantiene la calidad de obligado, como si el esclavo aún viviese. Aunque en este caso no se trata de una estipulación condicional, importa la doctrina del caso en cuanto la mora aparece como un elemento de juicio principal, a la hora de decidir la no extinción de la obligación en caso de morir el esclavo estipulado. Esta escueta formulación de Ulpiano no hace sino sintetizar la doctrina que antes había formulado Sabino, según la cual, si se estipulaba una cosa que ya era debida con anterioridad y respecto de la cual el promitente se encontraba en mora, aunque la cosa ahora estipulada pereciera, la acción nacida de la estipulación servía al estipulante para reclamar la prestación del deudor, la cual no se extinguía.

A partir de los testimonios tanto de Paulo como de Ulpiano, puede inferirse que la opinión sabiniana estimaba como regla general la inexistencia de acción en caso de perecer el esclavo antes de verificarse la prestación objeto de la obligación, sin distinguir si esta era condicional, o bien, pura y simple. Las excepciones a esta regla general residían en dos hipótesis: la primera, que el esclavo ahora adeudado hubiese sido exigible, con anterioridad a la estipulación, a través de la condictio; la segunda, que con anterioridad a la estipulación el esclavo hubiese sido adeudado, encontrándose el deudor en mora. En estas dos hipótesis la acción nacida de esta estipulación permite al estipulante reclamar la prestación adeudada, aunque el esclavo hubiese muerto.

\section{MARCELO Y ULPIANO}

Tanto Marcelo como posteriormente Ulpiano, vuelven a tratar el mismo caso del cual, según las noticias proporcionadas por Gayo, tratara en un primer momento Servio Sulpicio. Como se verá, ambos juristas coinciden en la misma doctrina, pudiendo decirse que la labor de Ulpiano se limita a una síntesis bastante fidedigna de lo que había afirmado Marcelo.

D. 46, 3, 72, 1 (Marcell. 20 dig.): Cum Stichum mibi deberes et in solvendo moram fecisses, sub condicione eum promisisti: pendente ea Stichus decessit: videamus, an, quia novari prior obligatio non potest, petitio servi competat ea, quae competeret, si non intercessisset stipulatio. sed in promptu contradictio est debitorem, cum stipulanti creditori sub condicione promisit, non videri in solutione hominis cessasse: nam verum est eum, qui interpellatus dare noluit, offerentem postea periculo liberari ${ }^{14}$.

\footnotetext{
13 Si el esclavo prometido hubiera muerto después de incurrir en mora quien lo prometió, queda este obligado, sin embargo, lo mismo que si viviera el esclavo.

${ }^{14}$ Debiéndome tú el esclavo Estico y habiendo incurrido en mora para pagarme, me lo has prometido bajo condición, y pendiente la condición ha muerto Estico: cabe preguntarse si, puesto que no pudo quedar novada la obligación anterior, no compete la misma acción por el esclavo que competiría si no hubiese
} 
Estamos ante una estipulación condicional (sub condicione eum promisisti) que se superpone a una obligación anterior ${ }^{15}$. En virtud de esta obligación primigenia, el promitente, que debía el esclavo Estico, no dio cumplimento a su obligación; por este motivo, al momento de realizar la estipulación condicionada, se encontraba en mora.

Interesa destacar, por ahora, que el estipulante y el originario deudor consisten en la misma persona; como veremos, no siempre es este supuesto idéntico. Frente a la cuestión de si se produce o no el efecto novatorio de la primera obligación, la respuesta del jurista viene a ser negativa. Aunque no se nos ha transmitido fundamento alguno para ello, este puede conjeturarse: Marcelo tenía dos opciones: o concedía efecto novatorio a la conclusión de la estipulación, o bien, al cumplimiento de la condición. Al negar la primera posibilidad, necesariamente aceptaba la segunda. Pero el hecho de la muerte del esclavo antes del cumplimiento de la misma le debió colocar ante otra interrogante: si al cumplirse la condición, la cosa estipulada había perecido ¿podía considerarse válida una estipulación carente de objeto? Marcelo debió tener ante sí otras dos posibilidades: o aceptaba un efecto retroactivo para la condición, o bien lo negaba. En otros términos: si la condición producía sus efectos hacia delante, no había posibilidad de dotar de validez a la estipulación, ya que esta no podía perfeccionarse, por carecer de objeto; si, en cambio, producía efecto retroactivo, se entendía que al momento de la conclusión la estipulación tenía objeto y era perfecta. Es lógico aceptar que Marcelo optó por la primera posibilidad. De ahí que la razón para negar efecto novatorio a la estipulación condicional haya dependido del hecho de haber muerto el esclavo antes de cumplirse la condición; de todo lo cual se extrae una conclusión: si el objeto de una estipulación sometida a condición perece antes del cumplimiento de la condición, impide que nazca acción para el estipulante (por no haber podido nacer la obligación) ${ }^{16}$. Pero, el elemento introducido por Marcelo, esto es, la mora del deudor en virtud del negocio primitivo, le plantea una nueva cuestión: ¿`tiene entonces el acreedor a su disposición la acción proveniente del primer negocio? Marcelo sostiene que aquí se produce una objeción que salta

habido estipulación; pero salta a la vista la objeción de que el deudor, al haber prometido bajo condición al acreedor estipulante, parece haber dejado de estar en mora, pues es verdad que el que no quiso pagar cuando se le interpeló se libera del riesgo al ofrecer luego el pago. Vid. KASER (1971) p. 256 n. 35, quien sorprendentemente cita este pasaje, a propósito de la continuidad de la exigibilidad de la obligación, una vez cumplida la condición después de la desaparición del objeto debido.

15 Parece discutible que el negocio estipulatorio superpuesto a la obligación anterior pueda calificarse de constitutum, ya que este consiste en un acuerdo por el cual el deudor (o un tercero) y el acreedor, aceptan que el pago de la deuda se realice dentro de un cierto plazo. Aunque la doctrina suele exigir el establecimiento de un plazo, a modo de elemento esencial, no es menos cierto que en las fuentes encontramos cuando menos un ejemplo en el cual no se conviene plazo, sino una condición; en tal caso, correspondería al acreedor una acción útil. Así, D. 13.5.19.1 (PAUL. 29 ad ed.): Sed is qui pure debet si sub condicione constituat, inquit Pomponius in hunc utilem actionem esse. [Si constituye bajo condición el que debe pura y simplemente, dice Pomponio que se puede dar contra él esta acción]. Vid. García Garrido (2004a) p. 245; GUZMÁN BRITO (1996) pp. 764 ss.

${ }^{16}$ La doctrina que aquí se atribuye a Marcelo fundamenta también otro responsum del mismo jurista:. D. 45.1 .98 pr. (MARCELL. 20 dig.)...et si fundi dominus sub condicione viam stipulatus fuerit, statim fundo alienato evanescit stipulatio. [...o si resulta que el dueño del fundo ha estipulado una servidumbre de paso, tan pronto como enajene aquel fundo, se desvirtúa la estipulación...] 
a la vista (sed in promptu contradictio est): el deudor, al estipular bajo condición ha dejado de estar en mora. El fundamento para ello, no puede ser más sencillo: el que habiendo sido interpelado para pagar no ha querido hacerlo, pero luego ofrece pagar, se libera del riesgo (qui interpellatus dare noluit, offerentem postea periculo liberari). Este motivo esgrimido por Marcelo solo puede querer decir lo siguiente: si al ofrecer el pago, después de haber incurrido en mora, el deudor deja de tener a su cargo el riesgo del perecimiento accidental de la cosa debida, entonces, la conclusión de la estipulación, a pesar de carecer de efecto novatorio, ha provocado un cambio en la carga del riesgo del perecimiento de la cosa, que se ha devuelto al acreedor, por haber eliminado, eso sí, un criterio siempre aceptado de transmisión del riesgo, es decir, la mora.

Ayudará a la mejor comprensión de cuanto aquí se dice, la exposición que viene a hacer Ulpiano de la doctrina de Marcelo.

D. 46. 2. 14 pr. (Ulp. 7 disput.): Quotiens quod pure debetur, novandi causa sub condicione promittitur, non statim fit novatio, sed tunc demum, cum condicio extiterit. et ideo si forte Stichus fuerit in obligatione et pendente condicione decesserit, nec novatio continget, quia non subest res eo tempore, quo condicio impletur. Unde Marcellus et si post moram Stichus in condicionalem obligationem deductus sit, purgari moram nec in sequentem deduci obligationem putat ${ }^{17}$.

Como se puede ver, Ulpiano expone los mismos supuestos de hecho que eran tratados en el responsum de Marcelo, a saber: i) obligación primigenia pura y simple, por la cual un esclavo es debido; ii) mora del deudor en entregar el esclavo; iii) nueva estipulación entre acreedor y deudor, sometida, en este caso, a condición; iv) muerte del esclavo antes del cumplimiento de la condición. A ello se suma la aceptación de la doctrina, según la cual, la segunda estipulación solo provocará un efecto novatorio en cuanto se cumpla la condición. De todo lo anterior, Ulpiano expone como consecuencia lógica (et ideo) que al producirse la muerte del esclavo antes del cumplimiento de la condición, esta quedará sin objeto al momento de cumplirse la condición. De acuerdo con lo dicho por Ulpiano, el responsum de Marcelo venía a establecer que la mora en entregar el esclavo, en la cual había incurrido el deudor en el cumplimiento de la obligación primigenia, quedaba ciertamente purgada y no se comprendía en la siguiente obligación ${ }^{18}$.

\footnotetext{
17 Cuando, a causa de la novación, se promete bajo condición lo que se debía sin condición, no se produce inmediatamente la novación, sino tan solo al cumplirse la condición. Así, pues, si la obligación tuviera por objeto Estico y este muriera pendiente la condición, no habrá novación, pues no subsiste la cosa en el momento de cumplirse la condición. Por esto piensa Marcelo que, si se hace una estipulación condicional de Estico cuando ya había incurrido en mora el deudor de la obligación anterior, queda purgada la mora y la responsabilidad por esta no entra ya en la nueva obligación.

18 Vid. KASER (1971) p. 516 n. 28. En mi opinión, la clasicidad de la respuesta de Marcelo encuentra su confirmación no solo en el hecho de ser citado por Ulpiano, sino en el más importante de que ambos textos pertenecen a masas diferentes -el de Ulpiano pertenece a la Sabiniana, en tanto el de Marcelo pertenece a la Edictal-, lo que difumina casi por completo, la posibilidad de la introducción de una interpolación.
} 
Desde la perspectiva de la comparación casuística, podemos extraer las siguientes que siguen.

El caso tratado inicialmente por Servio Sulpicio Rufo vuelve a ser tratado, poco más de un siglo después, por Marcelo. Este jurista adopta los mismos supuestos de hecho (identidad de debitum; estipulación condicional, muerte del esclavo), pero agrega una variante: la mora del deudor en el negocio originario. Sin embargo, los problemas acerca de los cuales debe dar su opinión son los mismos, es decir, el momento de la producción del efecto novatorio y los efectos para el acreedor de la muerte del esclavo antes del cumplimiento de la condición. El responsum de Marcelo introduce una opinión contraria a la mantenida por Servio Sulpicio, respecto del primer problema: la sola conclusión de la estipulación no ha podido producir el efecto novatorio respecto de la obligación emanada del negocio primitivo (quia novari prior obligatio non potest), el cual, a causa de la muerte del esclavo, no se producirá. A esta conclusión agrega otra, que sí viene a concordar en sus efectos con la sostenida por Servio: la acción anterior no subsistía, en razón de haber dejado de estar el riesgo a cargo del deudor. Esta opinión viene a ser confirmada por el testimonio que da Ulpiano acerca de la opinión de Marcelo. Por consiguiente, puede conjeturarse que ya a partir de Marcelo parece abandonarse la opinión de Servio Sulpicio, en el sentido de que la novación produjese efectos desde el momento de su conclusión, pero se mantiene el efecto de impedir al acreedor el ejercicio de la acción nacida del negocio originario. Si Servio no había dado fundamento para ello, Marcelo lo encuentra en el de la modificación de los criterios conforme a los cuales se atribuye el riesgo del perecimiento de la cosa debida.

\section{VENULEYO}

Centremos ahora la atención sobre Venuleyo, jurista contemporáneo de Marcelo y Juliano, quien nos proporciona una nueva redacción del caso que en su momento ocupara a Servio Sulpicio, Gayo y Marcelo.

D. 46.2.31 pr. (Ven. 3 de stip. ): Si rem aliquam dari stipulatus sum, deinde eandem sub condicione novandi animo ab eodem stipuler, manere oportet rem in rebus humanis, ut novationi locus sit, nisi si per promissorem steterit, quo minus daret. ideoque si hominem mihi dare te oporteat et in mora fueris, quo minus dares, etiam defuncto eo teneris: et si, priusquam decederet, cum iam mora facta sit, eundem a te sub condicione stipulatus fuero et servus postea decesserit, deinde condicio exstiterit, cum iam ex stipulatu obligatus es mibi, novatio quoque fiet ${ }^{19}$.

\footnotetext{
19 Si he estipulado que se me dé una cosa y luego la estipulo del mismo deudor con intención de novar y bajo condición, debe seguir existiendo la cosa para que tenga lugar la novación, a no ser que el promitente hubiera incurrido en mora. Así, pues, si me debes dar un esclavo y has dejado de dármelo por haber incurrido en mora, responderás aunque se haya muerto el esclavo y si hubiera estipulado de ti aquel esclavo bajo condición, antes de que muriera, pero después de haber incurrido tú en mora, luego muriera el esclavo, y entonces se cumpliera la condición, cuando ya me lo debías por estipulación, se produce también la novación.
} 
El fragmento que ahora nos ocupa trata del mismo caso que el de Marcelo. Sin embargo, hay matices que es necesario poner de relieve. En primer lugar, y, de cara a posibles críticas al texto, hay que hacer notar que el pasaje se inicia con una explicación de carácter general, a modo de un enunciado del cual deben extraerse deductivamente las consecuencias para cada caso. El tenor literal de esta parte del pasaje es algo oscuro y como introducción al caso que más adelante se resuelve, no resulta estrictamente coincidente. De acuerdo con esta explicación, si se estipula condicionadamente, con animus novandi $i^{20}$, para que se produzca el efecto novatorio, respecto de la misma obligación entre idénticas partes, se requiere que la cosa exista al momento de cumplirse la condición; tal subsistencia de la cosa no se exige si, al estipularse nuevamente, el deudor ya se encontraba en mora. Es decir, el jurista parece concentrar su atención en la existencia continuada del objeto de la estipulación condicional, para que esta, en definitiva, pueda producir su efecto novatorio; pero no deja lugar a dudas en cuanto a que el cumplimiento de la condición es un requisito igualmente indispensable para la producción de dicho efecto novatorio. En resumen, tratándose de una estipulación condicional, su efecto novatorio depende de dos requisitos: que la condición se cumpla y que la cosa objeto de la estipulación subsista hasta el momento de cumplirse la condición. Sin embargo, Venuleyo opina que no tienen lugar estas exigencias relativas al cumplimiento de la condición y a la subsistencia de la cosa debida hasta el evento antedicho, cuando el deudor de la nueva estipulación, ya en virtud de la obligación anterior, había incurrido en mora. Cabe preguntarse, entonces, cuál de los dos requisitos no se exige para la producción del efecto novatorio. Una posibilidad es que ninguno de los requisitos sea ahora exigible, o bien solo uno de ellos. Si se renuncia a exigir el cumplimiento de la condición, en tal caso, habría que decir que el efecto novatorio se produce desde el momento mismo de la conclusión del acto estipulatorio, de acuerdo con las alternativas que ya vimos a propósito de Marcelo. Sin embargo, no se ve la relación que existiría entre la mora de entregar el objeto prometido y el adelantamiento del efecto novatorio al momento de la conclusión de la novación. La otra posibilidad es que el único requisito que deje de exigirse sea el de la subsistencia del objeto hasta el cumplimiento de la condición. Es probable que la relación aquí sea más estrecha.

En este sentido, si se adelanta a este punto nuestra comparación casuística, se advierte que ya en esta primera parte del fragmento los supuestos de hecho son idénticos a los que enmarcaban los responsa de Servio Sulpicio y Marcelo (debitum originario; estipulación condicional, muerte del esclavo antes del cumplimiento de la condición); igualmente, los problemas planteados parecen haber sido los mismos, esto es, la determinación de la producción del efecto novatorio y los efectos de la muerte del esclavo antes

20 Esta expresión fue considerada insiticia por KRÜGER (1963) p. 795 n. 8, considera interpolada esta expresión, en lo cual sigue una tesis de Gradenwitz. A su turno, FUENTESECA (2000) p. 128 ss., realiza una meticulosa exposición de las posturas doctrinales de la Romanística desde el s. XIX. De dicha exposición, es posible observar cómo progresivamente la doctrina romanística va apartándose de los moldes de la crítica de interpolaciones, para llegar a resultados francamente anticríticos. Con todo, la autora (p. 142) es de la opinión de Bonifacio, según el cual al animus novandi no se le confirió relevancia alguna en la época clásica; ni siquiera a los últimos juristas clásicos es atribuible esta doctrina. 
del cumplimiento de la condición. Por último, una parte del responsum viene también a coincidir, en la medida que exige el cumplimiento de la condición para la producción del efecto novatorio. Pero los efectos de la muerte del esclavo reciben un tratamiento diferenciado. Venuleyo expresa que es necesario que el esclavo susbsista al momento de producirse la condición, de modo que cabe deducir que si perece antes del cumplimiento de esta, el deudor se libera de la obligación. Ambos razonamientos son coincidentes con los de Marcelo. Sin embargo, tratándose de la hipótesis de que el deudor, en virtud del negocio originario, ya se hubiese encontrado en mora de entregar el esclavo, Venuleyo introduce un parecer que contradice al de Marcelo, puesto que si este jurista consideraba que el deudor, al prometer nuevamente, aunque bajo condición, el esclavo debido, dejaba por este hecho de encontrarse en mora, Venuleyo sostiene, en cambio, que lo sigue debiendo, ya que no se exige que el esclavo subsista al momento del cumplimiento de la condición ${ }^{21}$. Esta mateira debió pues haberse convertido en un genuino ius controversum.

Veamos a continuación cómo explica el propio jurista en la segunda parte de este fragmento, cuanto ha mantenido en relación con el caso del esclavo adeudado. Venuleyo razona a través de dos hipótesis. La primera: que un esclavo sea debido, que el deudor se encuentre en mora en el cumplimiento de su prestación y que, durante la mora, el esclavo perezca (ideoque si hominem mibi dare te oporteat et in mora fueris, quo minus dares, etiam defuncto...). Esta hipótesis, que, como se ve, no trata de una obligación sometida ni a condición ni a término, sino más bien de una obligación pure, consiste solo en la exposición del problema del perecimiento de una cosa durante la mora del deudor. La respuesta de Venuleyo es que el deudor continuará obligado (eo teneris). O lo que es igual, que su obligación no se extingue. Pero si vamos más allá, observamos que en esta hipótesis Venuleyo ha seguido el parecer de Sabino, que antes examinábamos, que para la estipulación pura y simple de un objeto respecto del cual el deudor se encontraba ya en mora, concedía la acción nacida de la estipulación.

La segunda hipótesis que analiza Venuleyo, permite encajar este supuesto con los otros que hasta ahora han sido analizados: tomando el supuesto anterior del esclavo debido, y el hecho de encontrarse en mora el deudor, se agrega ahora el supuesto de que acreedor y deudor celebren una estipulación sometida a condición, en virtud de la cual se adeude el mismo esclavo. A ello se agregan dos hechos más: la muerte del esclavo antes del cumplimiento de la condición y el cumplimiento efectivo de esta (et si, priusquam decederet, cum iam mora facta sit, eundem a te sub condicione stipulatus fuero et servus postea decesserit, deinde condicio exstiterit, cum iam ex stipulatu obligatus es mihi). La respuesta de Venuleyo es que, dadas estas circunstancias, el efecto novatorio se produce de todos modos (novatio quoque fiet). Con este nuevo orden de cosas, quedan planteados todos los supuestos que habían enmarcado los responsa de Servio Sulpicio y Marcelo; quedan en pie los problemas sobre los cuales estos habían debido responder y queda, finalmente, un responsum que solo da cuenta del problema de la producción del efecto novatorio. Venuleyo, por tanto, no dice expresamente qué efecto, de cara a las

${ }^{21}$ Vid. Pennitz (2000) p. 213 n. 90. 
acciones del acreedor, produce la muerte del esclavo. Sin embargo, de su respuesta puede colegirse la correspondiente a esta segunda cuestión: si con el cumplimiento de la condición se produce el efecto novatorio, entonces es evidente que la acción nacida del negocio originario ya no se encuentra a disposición de acreedor, puesto que se ha extinguido ipso iure. Luego ¿dispone el acreedor de la acción nacida de la estipulación novatoria, contra el deudor, a pesar de que el esclavo haya premuerto? Todo parece indicar que sí, ya que no tendría sentido hablar de efecto novatorio de una estipulación en virtud de la cual no fuese posible ejercitar la acción correspondiente.

Pero cabe ahora preguntarse por el fundamento de este responsum. La primera posibilidad es que este haya de buscarse en el efecto retroactivo de la condición cumplida. Así -podría argumentarse- de cumplirse la condición, se entiende nacida la obligación al momento de la conclusión de la estipulación, por lo que la muerte posterior del esclavo, estaría a cargo del promitente. Pero, en tal caso, sería necesario justificar por qué motivo no se concede idéntico efecto retroactivo a la condición, cuando el objeto vendido no se encontraba en mora de ser entregado, como ocurre con la primera parte del fragmento, en que Venuleyo exige la subsistencia de la cosa debida al momento de cumplirse la condición. Sería necesario aquí establecer una estrecha relación entre la mora y el efecto retroactivo de la condición, pero esta no alcanzo a verla. Por este motivo, pienso que la justificación deberá encontrarse en otro argumento. Este podría ser el siguiente: si Venuleyo quería ofrecer una opinión disidente de la de Marcelo, era lógico que discutiese los fundamentos de la solución. Marcelo había invocado como fundamento de su decisión el argumento según el cual, si después de encontrarse en mora, el deudor ofrecía el pago (a través de una estipulación sometida a condición), entonces no soportaba el riesgo por el perecimiento de la cosa. Venuleyo, al dictaminar en sentido contrario, lo que hace es atacar, precisamente, este argumento: en virtud de la estipulación no queda purgada la mora, por lo que el riesgo seguía estando a cargo del deudor. Luego, la doctrina de este último jurista viene a estar asentada no en una perspectiva diferente de los efectos de la mora respecto de su incidencia en el riesgo que debe asumir el deudor, sino en la ausencia de efecto purgatorio de la mora de la estipulación condicional. Mirado desde la perspectiva del acreedor, ningún beneficio habría obtenido si, a más de serle ahora debida la cosa en virtud de estipulación, nuevamente traía sobre sí la carga del riesgo, si es que el esclavo moría antes del cumplimiento de la condición. La decisión de Venuleyo permite equilibrar los beneficios de la nueva estipulación; por una parte, el deudor no queda obligado sino a partir del cumplimiento de la condición; pero en la medida que obtiene este beneficio, mantiene la carga de soportar el riesgo del perecimiento del esclavo, antes de cumplimiento de la condición.

La comparación entre estos fragmentos arroja las conclusiones que se exponen a continuación. Venuleyo parte de los mismos supuestos que Servio Sulpicio y Marcelo, con excepción de la mención del animus novandi de la estipulación superpuesta, que no aparece en los juristas anteriores; los problemas que se le plantean son, asimismo, idénticos. Al llegar a la respuesta, Venuleyo incorpora una diferencia, tratándose de la cuestión de la purga de la mora por la nueva estipulación. Este jurista declara aceptar el hecho de que el efecto novatorio depende no solo del cumplimiento de la condición, sino además, 
de la pervivencia del esclavo hasta el cumplimiento de la condición antedicha, tal como Marcelo seguramente debió también creer justificadamente. Sin embargo, piensa además que si el deudor ha incurrido en mora, tratándose del negocio originario, la subsistencia del esclavo hasta el cumplimiento de la condición perdía su importancia, de cara al cumplimiento de la condición, por lo cual, ya no era exigible como requisito para la producción del efecto novatorio, una vez cumplida la condición. La fundamentación de esta parte del responsum no nos ha sido transmitida, si es que ella efectivamente existió. Solo queda conjeturar que en Venuleyo, los efectos de la mora, de cara a la carga del riesgo, no se extinguían por el hecho de concluirse una nueva estipulación, aunque esta tuviese después carácter novatorio. Luego, en este jurista se acusa la introducción de dos nuevos criterios que enriquecen la discusión: el animus novandi y la negación de la purga de la mora, en virtud de la conclusión de la estipulación condicional.

Tanto este texto como el anterior resultan a juicio de Haymann ${ }^{22}$ suficientes testimonios del modo en que un clásico abordaba el problema del efecto retroactivo de las condiciones. Luego, al no observar un tratamiento igual de cuidadoso por parte de Paulo, termina por conjeturar su carácter insiticio.

\section{JULIANO}

Una nueva redacción del caso la presenta Juliano. Como es previsible, Juliano varía en alguna medida los supuestos, pero, como veremos, resulta fácilmente reconocible la identidad del caso tratado con la que hasta ahora hemos venido examinando en los otros juristas.

D. 45.1 .56 .8 (Iul. 52 dig.): Si hominem, quem a Titio pure stipulatus fueram, Seius mihi sub condicione promiserit et is pendente condicione post moram Titii decesserit, confestim cum Titio agere potero, nec Seius existente condicione obligetur: at si Titio acceptum fecissem, Seius existente condicione obligari potest. [idcirco haec tam varie, quod homine mortuo desinit esse res, in quam Seius obligaretur: acceptilatione interposita superest homo, quem Seius promiserat $]^{23}$.

En el caso planteado por Juliano, el contexto viene dado por los siguientes supuestos: un esclavo era debido por Ticio, en virtud de una estipulación pura y simple (pure stipulatus), respecto del cual el promitente (Ticio) había incurrido en mora. Encontrándose, pues, incumplida la obligación de Ticio a causa de la mora, Seyo promete al acreedor de Ticio, bajo condición el mismo esclavo debido; posteriormente y encontrán-

22 Haymann (1920) p. 82.

23 Si Seyo me prometiera bajo condición dar un esclavo que yo había estipulado sin ella de Ticio, y este esclavo se muriera pendiente la condición, después de haber incurrido Ticio en mora, podré reclamar inmediatamente de Ticio, y Seyo no quedará obligado ni aun después de cumplirse la condición; pero si Ticio hubiese aceptilado conmigo, puede quedar obligado Seyo al cumplirse la condición. La diferencia está en que, si se muere el esclavo, deja de existir el objeto de la obligación de Seyo, en tanto, si se interpone aceptilación, subsiste siempre el esclavo que Seyo había prometido. 
dose aún pendiente la condición, el esclavo muere (et is pendente condicione post moram Titii decesserit). La primera cuestión que se plantea a Juliano es la siguiente: ¡a quién debe demandar el acreedor, a Ticio o a Seyo? La respuesta de Juliano es que puede demandar a Ticio de inmediato (confestim), puesto que Seyo, aun cuando la condición se cumpliese, no podría quedar obligado (nec Seius existente condicione obligetur).

Toda esta primera parte tiene la virtud de plantear, de una vez, todos los supuestos del caso y, a continuación, la solución a este. Si se analizan los términos del reponsum el fundamento de este parece ser el siguiente: al decir Juliano que si el esclavo muere antes del cumplimiento de la condición, Seyo no quedará obligado, aunque posteriormente la condición se cumpla, parece excluir de la discusión el efecto retroactivo de la condición. En efecto, es de toda lógica pensar que si se quisiere sostener la opinión contraria, la retroactividad de la condición sería el fundamento más adecuado. Ahora bien, el adverbio confestim, con el cual Juliano expresa que nada más morir el esclavo antes de cumplirse la condición puede el acreedor dirigirse contra el primer deudor, esto es, Ticio, permite conjeturar una diferencia con el parecer de Servio Sulpicio, en cuanto al momento de la producción del efecto novatorio por parte de la nueva estipulación. En el fondo del razonamiento de Juliano se encontraría también la idea de que la novación debiera ser un efecto del cumplimiento de la condición.

Si nos fijamos ahora en la segunda parte del texto, vemos que este viene presidido por la conjunción adversativa $a t$, lo cual quiere decir que necesariamente encontraremos una solución opuesta a la que se acaba de estudiar. En otras palabras, se quiere mentar una excepción a cuanto se ha dicho. Esta excepción consiste en decir que si el acreedor hubiese liberado a Ticio (Titio acceptum fecissem), al cumplirse la condición Seyo quedaría obligado (Seius existente condicione obligari potest). Resulta pertinente, llegados a este punto, preguntarse qué circunstancias son las que contradice esta excepción a la regla. Una primera opción es entender lo siguiente: que si el esclavo muere antes del cumplimiento de la condición, Seyo resulta igualmente obligado. Siendo esto así, deberemos buscar las razones que justifiquen una decisión de esta naturaleza. La primera podría encontrarse en el efecto retroactivo de la condición. Pero, al igual que lo que ocurría con Venuleyo, obstaría a esta explicación el sencillo hecho de que Juliano no asigna idéntico efecto a la condición, cuando Ticio no ha sido liberado. Una segunda razón podría ser la siguiente: Seyo se ha hecho cargo de un debitum, cuya cosa ya se encontraba en mora y esta situación ha condicionado que Seyo deba responder por la cosa, incluso si esta desaparece antes de cumplirse la condición. Dicho de otra manera, esto vendría a querer decir que la mora es una circunstancia que queda excluida de los términos de la liberación de Ticio, por lo cual agrava la situación de Seyo. Me parece este un argumento carente de sentido y que no encuentra asidero en el contexto del pasaje.

Una segunda opción es pensar que no se ha referido Juliano con esta excepción a cada uno de los supuestos del fragmento, sino que ha querido, sencillamente, oponer este razonamiento al que le era inmediatamente anterior, esto es, al argumento según el cual nec Seius existente condicione obligetur. Juliano solo habría querido decir que tratándose de un negocio novatorio por cambio de deudor, al quedar liberado el antiguo deudor, tendría el acreedor para cobrar su crédito solo al nuevo deudor y esto, una vez 
que la condición se haya cumplido. En cuanto a la muerte del esclavo antes del cumplimiento de la condición, pienso que esta es una circunstancia que también queda excluida de la hipótesis que preside esta solución, con lo cual, creo que la adversativa at debe entenderse solo respecto de la afirmación según la cual, ni cumpliéndose la condición se obliga Seyo.

Mi crítica a lo expresado por Juliano, a partir de idcirco encuentra su fundamentación en el siguiente orden de ideas. Si nos fijamos en el tenor literal de esta parte del fragmento, ella expresa lo siguiente: si muere el esclavo, deja de existir el objeto de la obligación de Seyo, pero si se ha interpuesto aceptilación, subsiste siempre el esclavo que Seyo había prometido. A mi juicio, hay aquí mucha oscuridad y por ello me detendré un poco más en esta argumentación.

El criterio según el cual, de morir el esclavo deja de existir el objeto de la obligación de Seyo y por tanto no puede este obligarse (quod homine mortuo desinit esse res, in quam Seius obligaretur), no es nuevo, ya que hemos visto también a Venuleyo expresarse de modo parecido, en relación con la producción del efecto novatorio (manere oportet rem in rebus humanis, ut novationi locus sit) y lo hemos presupuesto en Marcelo. Se trata, por tanto, de un requisito que, a pesar de los matices con que era expresado, apuntaba siempre a la subsistencia del objeto prometido al momento de cumplirse la condición y habla, por tanto, de un cierto ius constitutum acerca de esta cuestión. Desde este punto de vista, puede afirmarse que la respuesta a la primera pregunta que se plantea a Juliano se encuadra perfectamente dentro de lo que los juristas del Principado habían venido aceptando. En nuestra opinión, la respuesta a la cuestión planteada por la introducción de la aceptilación de la deuda de Ticio, resulta sorprendente. En efecto, lo que viene a decir el texto de Juliano es que cuando la deuda anterior ha sido cancelada, el esclavo subsiste hasta el cumplimiento de la condición, aunque muera antes de cumplirse esta, lo cual, evidentemente, carece de $\operatorname{sentido}^{23}$. En mi opinión, toda esta última parte (idcirco-fin) no es más que un glosema, bastante torpe por lo demás, ya que introduce un incongruente e insostenible argumento, como lo es la (¿fingida?) subsistencia del esclavo y, junto con ello, no da buena cuenta de los motivos que Juliano tuvo para conceder la acción en contra de Seyo. Si, por otra parte, se compara esta solución con la dada por Venuleyo, respecto del esclavo cuya entrega el promitente había demorado -supuesto diferente, por tanto- resulta que la solución sigue siendo la misma: aunque el esclavo ha premuerto al cumplimiento de la condición, el promitente queda igualmente obligado.

Desde el punto de vista de la comparación entre fragmentos, pueden ser extraídas las siguientes conclusiones: el jurista mantiene el análisis dentro del marco de los supuestos de hecho básicos, que han caracterizado al caso-guía, a saber: i) que en el cumplimiento de la obligación anterior el deudor había incurrido en mora, ii) que la nueva estipulación se encuentra sometida a condición y iii) que el esclavo muere antes

${ }^{24}$ En opinión de Mollá NebOT (1993) p. 167, el periodo comprendido entre at y obligari potest puede estar incompleto, ya que no contempla todas las posibilidades. Una de estas sería el supuesto de aceptilación de la obligación condicional de Seyo. 
de cumplirse la condición. De los problemas planteados a partir de dicho caso-guía, Juliano solo responde con claridad acerca de uno de ellos, es decir, el relativo a la subsistencia de la acción a favor del acreedor; sin embargo, de su respuesta puede colegirse su postura frente al problema del efecto novatorio. A juicio de Juliano, este solo se produce una vez cumplida la condición. La elucidación de este problema se vincula con la variante que introduce Juliano al caso, en relación con las personas que intervienen en los actos. Si en el caso resuelto por Servio, Marcelo y después Venuleyo, las partes, tanto del negocio originario como de la estipulación superpuesta, eran las mismas, Juliano introduce la modificación de que tratándose de esta última estipulación el promitente del esclavo sea una persona distinta del primer deudor. Por esta razón, las consecuencias toman en cuenta la figura de uno y otro deudor. El responsum julianeo distingue dos situaciones: la primera, que la obligación primigenia no haya sido extinguida en virtud de algún acto formal, como la aceptilación; la segunda es que este acto formal de extinción sí haya tenido lugar. En el primer caso, el acreedor podrá dirigirse en contra del primer deudor, valiéndose para ello de la acción emanada del negocio originario; en el segundo caso, si la condición se cumple, el efecto novatorio se habrá producido de todos modos, por lo que el promitente se verá obligado en virtud de la acción nacida de la estipulación.

\section{SÍNTESIS ESQUEMÁTICA}

Quisiera ofrecer a continuación un esquema de cuanto se ha analizado, a propósito de la redacción de un mismo caso por diferentes juristas. Ello permite, en mi opinión, contemplar con mayor claridad los puntos de coincidencias y las diferencias de cada uno. Pienso que ello contribuye a establecer con mayor claridad lo que ha sido mi tesis en este punto: que se trata de un mismo caso y que es posible observar en él variantes del mismo caso, según la exposición de cada jurista. Esta exposición precederá a las conclusiones a las cuales creo posible llegar, a partir de este examen.

\begin{tabular}{|c|c|c|c|}
\hline JURISTA & SUPUESTOS & PROBLEMAS & RESPUESTAS \\
\hline \multirow{2}{*}{$\begin{array}{l}\text { Servio } \\
\text { Sulpicio } \\
\text { Rufo }\end{array}$} & \multirow{2}{*}{$\begin{array}{l}\text { - Debitum originario } \\
\text { - Estipulación condicio- } \\
\text { nal superpuesta } \\
\text { - Muerte del esclavo antes } \\
\text { de cumplimiento de la } \\
\text { condición }\end{array}$} & $\begin{array}{l}\text { - Momento del efecto no- } \\
\text { vatorio }\end{array}$ & $\begin{array}{l}\text { - Desde conclusión de es- } \\
\text { tipulación }\end{array}$ \\
\hline & & $\begin{array}{l}\text { Efecto de la muerte del } \\
\text { esclavo respecto de las } \\
\text { acciones del estipulante. }\end{array}$ & $\begin{array}{l}\text { - El acreedor carece de ac- } \\
\text { ción }\end{array}$ \\
\hline Sabino & $\begin{array}{l}\text { - Estipulación condicio- } \\
\text { nal }\end{array}$ & $\begin{array}{l}\text { Efecto del perecimiento } \\
\text { de la cosa adeudada sin } \\
\text { mora del deudor }\end{array}$ & $\begin{array}{l}\text { - Si el deudor no se en- } \\
\text { contraba en mora: extin- } \\
\text { ción de deuda } \\
\text { - Si el deudor se encontra- } \\
\text { ba en mora: subsistencia } \\
\text { de obligación }\end{array}$ \\
\hline
\end{tabular}




\begin{tabular}{|c|c|c|c|}
\hline JURISTA & SUPUESTOS & PROBLEMAS & RESPUESTAS \\
\hline \multirow[t]{2}{*}{ Marcelo } & \multirow{2}{*}{$\begin{array}{l}\text { - Debitum originario } \\
\text { - Mora del deudor } \\
\text { - Estipulación condicio- } \\
\text { nal superpuesta } \\
\text { - Muerte del esclavo antes } \\
\text { del cumplimiento de la } \\
\text { condición }\end{array}$} & $\begin{array}{l}\text { - Momento del efecto no- } \\
\text { vatorio }\end{array}$ & $\begin{array}{l}\text { - Cumplimiento de la con- } \\
\text { dición }\end{array}$ \\
\hline & & $\begin{array}{l}\text { Efecto de la muerte del } \\
\text { esclavo antes del cum- } \\
\text { plimiento de la condi- } \\
\text { ción, respecto de las ac- } \\
\text { ciones del estipulante }\end{array}$ & $\begin{array}{l}\text { - El acreedor no puede re- } \\
\text { clamar por la mora }\end{array}$ \\
\hline \multirow[t]{4}{*}{ Venuleyo } & \multirow{2}{*}{$\begin{array}{l}\text { - Debitum originario } \\
\text { Estipulación condicio- } \\
\text { nal novatoria (animus } \\
\text { novandi) }\end{array}$} & $\begin{array}{l}\text { - Momento del efecto no- } \\
\text { vatorio }\end{array}$ & $\begin{array}{l}\text { - Cumplimiento de la con- } \\
\text { dición }\end{array}$ \\
\hline & & $\begin{array}{l}\text { Efecto de la muerte del } \\
\text { esclavo antes del cum- } \\
\text { plimiento de la obliga- } \\
\text { ción respecto de las ac- } \\
\text { ciones del estipulante }\end{array}$ & $\begin{array}{l}\text { - Si el promitente no se } \\
\text { encontraba en mora: ex- } \\
\text { tinción de la deuda } \\
\text { - Si el promitente se en- } \\
\text { contraba en mora: sub- } \\
\text { siste la obligación }\end{array}$ \\
\hline & $\begin{array}{ll}\text { - } & \text { Esclavo debido } \\
\text { - } & \text { Mora del deudor } \\
\text { - } & \text { Muerte del esclavo du- } \\
& \text { rante la mora } \\
\end{array}$ & $\begin{array}{l}\text { Efecto de la muerte del } \\
\text { esclavo, respecto de sub- } \\
\text { sistencia de la obliga- } \\
\text { ción }\end{array}$ & $\begin{array}{l}\text { - Deudor continúa obliga- } \\
\text { do }\end{array}$ \\
\hline & $\begin{array}{l}\text { - Debitum originario } \\
\text { - Mora del deudor } \\
\text { - Estipulación condicio- } \\
\text { nal superpuesta } \\
\text { - Muerte del esclavo antes } \\
\text { de cumplimiento de la } \\
\text { condición }\end{array}$ & $\begin{array}{l}\text { Momento del efecto no- } \\
\text { vatorio } \\
\text { Efecto de la muerte del } \\
\text { esclavo, respecto de la } \\
\text { subsistencia de la obli- } \\
\text { gación }\end{array}$ & $\begin{array}{l}\text { Cumplimiento de la con- } \\
\text { dición } \\
\text { - Deudor continúa obliga- } \\
\text { do. }\end{array}$ \\
\hline \multirow[t]{2}{*}{ Juliano } & \multirow{2}{*}{$\begin{array}{l}\text { - Debitum originario } \\
\text { - Estipulación condicio- } \\
\text { nal superpuesta. } \\
\text { - Variante: la persona del } \\
\text { promitente no es el deu- } \\
\text { dor originario, sino un } \\
\text { tercero. } \\
\text { - Muerte del esclavo antes } \\
\text { de cumplimiento de la } \\
\text { condición }\end{array}$} & $\begin{array}{l}\text { - Momento del efecto no- } \\
\text { vatorio }\end{array}$ & $\begin{array}{l}\text { - Cumplimiento de la con- } \\
\text { dición }\end{array}$ \\
\hline & & $\begin{array}{l}\text { - Efectos de la muerte del } \\
\text { esclavo, respecto de la } \\
\text { subsistencia de la obli- } \\
\text { gación }\end{array}$ & $\begin{array}{l}\text { - Si el acreedor no aceptiló } \\
\text { al primer deudor, hay ac- } \\
\text { ción contra este, pero no } \\
\text { contra el segundo. } \\
\text { - Si hubo aceptilación del } \\
\text { primer deudor, hay ac- } \\
\text { ción contra el segundo } \\
\text { deudor. }\end{array}$ \\
\hline Gayo & $\begin{array}{l}\text { - Debitum originario } \\
\text { - Estipulación condicio- } \\
\text { nal } \\
\text { - Muerte del esclavo antes } \\
\text { del cumplimiento de la } \\
\text { condición }\end{array}$ & $\begin{array}{l}\text { Momento del efecto no- } \\
\text { vatorio } \\
\text { Efectos de la muerte del } \\
\text { esclavo, respecto de la } \\
\text { subsistencia de la obli- } \\
\text { gación }\end{array}$ & $\begin{array}{l}\text { - Cumplimiento de la con- } \\
\text { dición } \\
\text { - El promitente dispone } \\
\text { de excepciones contra la } \\
\text { acción del estipulante }\end{array}$ \\
\hline
\end{tabular}




\section{CONCLUSIONES}

A lo largo de este trabajo he tratado de defender la tesis de que tanto juristas pertenecientes tanto a la jurisprudencia republicana como a la clásica, se ocuparon de un mismo caso que trataba del perecimiento de la cosa objeto de la prestación de una estipulación condicional, al cual iban introduciendo variantes que les permitían extraer diversas soluciones. Creo haber probado suficientemente esta redacción sucesiva del mismo caso, identificando cada una de las variantes. A partir del examen de todos estos casos, creo que es posible rescatar algunas conclusiones.

Para Servio Sulpicio Rufo, dada una obligación condicional, si perecía la cosa objeto de la prestación del deudor antes de verificarse el cumplimiento de la condición, quedaba el acreedor impedido de reclamar el cumplimiento de la prestación. Ciertamente, en un negocio formal como es la stipulatio, en que solo el promitente quedaba gravado con una obligación, esta decisión impedía al estipulante reclamar lo adeudado, por lo menos en virtud de la estipulación condicional. Luego, en un jurista republicano como Servio, no se concedía a la condición cumplida efecto retroactivo. Si así hubiere efectivamente ocurrido, el acreedor habría dispuesto de una acción nacida de la estipulación condicional contra el deudor, pero, como se ve, no es esta la situación. Tampoco Gayo concedió este efecto retroactivo a la condición cumplida. En este sentido, no debe confundirnos el hecho de que Gayo y Servio mantengan posiciones encontradas acerca de la necesidad del cumplimiento de la condición para la producción del efecto novatorio, ya que esta es una cuestión que solo atañe a la extinción de la obligación anterior, pero que no prejuzga acerca de la concesión de la acción nacida de la nueva estipulación al acreedor. En este mismo sentido, es necesario tener claro que cuando Gayo se refiere a las excepciones oponibles al acreedor, lo hace en el entendido de que este ejercita la del negocio originario, en razón de que el efecto novatorio no llegó a producirse.

Si se toma en cuenta ahora la opinión de Marcelo, puede observarse que este jurista sostiene una media sententia, acerca de lo afirmado por Servio. En efecto, aunque Marcelo piensa que el efecto novatorio solo se produce una vez cumplida la condición, de todos modos excluye la posibilidad de que el acreedor pueda ejercitar una acción que incluya la responsabilidad del deudor por la mora en que había incurrido, respecto del negocio originario. La fundamentación que veíamos de Marcelo, relacionaba directamente el tema de la mora con el riesgo asumido por el deudor. En pocas palabras, venía a decir que, si había dejado de estar en mora, en razón de la nueva estipulación condicional, entonces, ya no soportaba el riesgo del perecimiento. Tal como se ha visto, Marcelo es el único jurista que de modo claro e inequívoco formula esta estrecha relación entre mora y riesgo, a propósito del cumplimiento de la prestación emanada de una estipulación, pero se trata de una constante en el pensamiento de los juristas. Por otra parte, el problema del cumplimiento de la condición después del perecimiento del esclavo no es abordado por Marcelo. Pero puede conjeturarse que no habría modificado la opinión del jurista; en efecto, el solo hecho de mencionar la muerte del esclavo como el único factor de la no producción del efecto novatorio, hace pensar que no necesitaba el jurista constatar el posterior cumplimiento o frustración de la condición; el silencio 
sobre estas cuestiones bien puede interpretarse como un indicio del poco valor que el jurista le asignaba. Luego, si el cumplimiento de la condición no alteraba sustancialmente la no producción del efecto novatorio, cabe entonces deducir que el cumplimiento de dicha condición carecía de efecto retroactivo. De ahí que me interese destacar que también en este jurista no cabía plantearse siquiera si el cumplimiento de la condición podía producir un efecto retroactivo.

Si se examina, a continuación, la solución propuesta por Venuleyo para el mismo caso, se observa que solo en este jurista se produce el efecto novatorio a partir del cumplimiento de la condición, no obstante haber premuerto el esclavo objeto de la prestación. Pero, como se ha visto, no debe necesariamente verse ahí un efecto retroactivo de la condición. La razón que he esgrimido para ello es que si el deudor no se hubiese encontrado en mora, habría carecido el acreedor de acción para reclamar, aunque sí se hubiese producido el cumplimiento de la condición. Por tanto, se descarta también en Venuleyo el efecto retroactivo de la condición.

A su turno, el mismo caso, ahora estudiado por Juliano, ha llevado a una solución parecida a la examinada en Venuleyo. Hemos detectado, por una parte, que en Juliano se mantiene la idea de que para que la prestación nacida de una obligación condicional exista, debe el objeto de aquella subsistir hasta el momento del cumplimiento de la condición. Ello, pienso, da suficientemente cuenta del desconocimiento en un jurista como Juliano del efecto retroactivo de la condición, puesto que, de haberlo conocido, su argumentación en este sentido sería incongruente.

Por tanto, podría sostenerse que para un sector importante de la jurisprudencia republicana y clásica, hay dos afirmaciones importantes que pueden ser hechas. La primera: un supuesto de hecho habitual, tratándose de obligaciones condicionales, fue el perecimiento de la cosa objeto de la prestación, antes del cumplimiento de la condición. La segunda: en relación con los efectos que dicho perecimiento provocaba, no asignó la jurisprudencia efecto retroactivo al cumplimiento de la condición.

Una parte de la romanística ha tratado de conectar la doctrina emanada de algunos de estos pasajes con la exégesis de D. 18.6.8 pr. Bauer ${ }^{25}$ piensa que los tres juristas citados por Paulo aprobaban un efecto ex tunc del cumplimiento de la condición, es decir, retroactivo. Así, el traspaso del riesgo solo se verificaría ya al momento de la conclusión del contrato, ciertamente si en su momento la condición se hubiese cumplido. Paulo habría mantenido una opinión distinta; más concretamente, la contraria. Para sostener esta conclusión, Bauer se apoya en Gai. 3. 179, que, según su exégesis, muestra cómo Gayo opinaba que la condición producía sus efectos ex nunc, en contra de lo que pensaba Servio, quien habría aceptado que la condición producía sus efectos ex tunc, es decir, retroactivo. Las consecuencias que de todo esto extrae Bauer son del todo lógicas: en primer lugar, la retroactividad del cumplimiento de la condición debió ser conocida en el derecho que denominamos republicano; en segundo lugar, que todavía en el derecho clásico, algún rol desempeñaba, puesto que de lo contrario, Gayo no habría necesitado contraponer ambas opiniones.

25 BAUER (1998) p. $32 \mathrm{~s}$. 
Por el contrario, Guzmán Brito ${ }^{26}$, plantea la imposibilidad de colocar en un mismo nivel los efectos de la condición en una estipulación y en un contrato consensual. Habría una diferencia fundamental: en la estipulación condicional la obligación se considera nacida en el momento en que ha sido celebrada la estipulación (o sea, ex tunc), todo lo cual es debido al carácter formal de la estipulación. O dicho de otro modo: la condición suspende el efecto obligacional, pero no la estipulación misma, que existe desde el primer momento. Así, una vez cumplida la condición, el efecto debe ser atribuido de modo directo e inmediato a la estipulación preexistente: la causa de la obligación, por tanto, no es el hecho en que consistía la condición, sino la estipulación. Por el contrario, en los negocios consensuales, la condición viene a suspender el negocio mismo y este solo se considera existente una vez cumplida la condición; por tanto, esta nace ex tunc. Por ello, piensa Guzmán, es erróneo plantear este problema en términos de efecto retroactivo: no se trata de si la condición produce o no tal efecto (que no lo produce), sino de determinar en qué momento surge el negocio mismo, que al único que puede imputarse un efecto obligacional. La disparidad de soluciones ofrecidas por las fuentes debe achacarse a la disparidad de negocios y a la inexistencia de un principio general sobre la materia.

\section{BIBLIOGRAFÍA CITADA}

BAUER, Martin (1998): Periculum emptoris. Eine dogmengeschichtihe Untersuchung zur Gefahrtragung beim Kauf(Berlin, Dunker \& Humblot) 283 pp.

Castresana Herrero, Amelia (1994): "Las estipulaciones", Derecho Romano de Obligaciones. Homenaje al Profesor José Luis Murga (Madrid, Editorial Centro de Estudios Ramón Areces S.A.) pp. 439-462.

FUENTESECA, Margarita (2000): El problema de la relación entre 'novatio' y 'delegatio' desde Roma hasta las codificaciones europeas (Madrid, Dykinson) 282 pp.

GarCía GARRIDO, Manuel (1987): "Redacciones coincidentes (leges geminatae) y casos jurisprudenciales semejantes (capita similia)", en: Estudios de Derecho romano en honor de Álvaro d'Ors (Pamplona, EUNSA, tomo I) pp. 517-537.

GARCÍA GARRIDO, Manuel (1988): "Realidad y abstracción en los casos jurisprudenciales romanos” en: Estudios en homenaje al profesor Juan Iglesias” (Madrid, Universidad Complutense de Madrid, tomo I) pp. 249-261.

García Garrido, Manuel (2004a): Derecho Privado Romano (Madrid: Ediciones Académicas S.A., 14a ed.) 500 pp.

García Garrido, Manuel (2004b): Casuismo y jurisprudencia (Madrid, Ediciones Académicas S.A., 2 edición) 611 pp.

GuZMÁn BRITO, Alejandro (1996): Derecho Privado Romano (Santiago, Editorial Jurídica de Chile, tomo I).

${ }^{26}$ GUZMÁN BRITO (1996) p. 37. 
Haymann, Franz (1920): “Textkritische Studien zum römischen Obligationenrecht II. Periculum est emptoris", Zeitschrift der Savigny-Stiftung. Romanistische Abteilung No 41: pp. 44-185.

Kaser, Max (1971): Römisches Privatrecht, 2a ed. (München, Beck, tomo I).

KRÜGER, Paul (1963): Iustiniani Digesta (Weidmann, Berlín, 13a edición) 957 pp.

LAZO, Patricio (2005): "Dos aspectos de la perfección de la compra en la doctrina de Paulo", Anuario de la Facultad de Ciencias Juridicas de la Universidad de Antofagasta, No 10 (en prensas).

MitTeIS, Ludwig (1994): Römisches Privatrecht bis auf die Zeit Diokletians, reimpresión de la edición de 1908 (Aalen, Scientia) 428 pp.

Mollá Nebot, Sonia (1993): Extinción formal de las obligaciones verbales. La "acceptilatio" (Madrid, Civitas) 250 pp.

D’Ors, Álvaro (1986): Derecho Privado Romano (Pamplona, EUNSA) 635 pp.

PENNITZ, Martin (2000): Das 'periculum rei venditae'. Ein Beitrag zum ,aktionenrechtlichen Denken “ im römischen Privatrecht (Wien-Köln-Weimar, Böhlau) 512 pp.

SECKEL, Emil y LEVY, Ernst (1927): "Die Gefahrtragung beim Kauf im klassischen römischen Recht", Zeitschrift der Savigny-Stiftung. Romanistische Abteilung No 47: pp. 117-263.

TAlamanCA, Mario (1990): Istituzioni di diritto romano (Milano, Giuffrè) 829 pp. 
